\title{
La dieta vegetariana en niños y preadolescentes
}

\author{
Vegetarian diet in children and pre-adolescents
}

Daniela Belen Arona ${ }^{\mathrm{a}}$, Guillermo Gregorio Pietropaolo ${ }^{\mathrm{a}}$, Juan Víctor Ariel Franco $\mathrm{b}^{\mathrm{b}, \mathrm{c}, \mathrm{d}}$

\begin{abstract}
Resumen
Basándonos en una consulta de una niña que desea realizar una dieta vegetariana se realizó una búsqueda bibliográfica para identificar evidencia sobre el impacto de este tipo de dieta en la salud. La evidencia actual indica que la dieta vegetariana es una opción saludable que no produciría un impacto negativo en el crecimiento y desarrollo de los niños, si bien existiría la posibilidad de déficit de micronutrientes.
\end{abstract}

\begin{abstract}
Based on a consultation of a girl who wishes to make a vegetarian diet, a literature search was conducted to identify evidence on the impact of this type of diet on health. Current evidence indicates that the vegetarian diet is a healthy option that would not have a negative impact on the growth and development of children, although there is a possibility of micronutrient deficits.
\end{abstract}

Palabras clave: Dieta vegetariana, crecimiento, desarrollo, niños, adolescentes. Keywords: Vegetarian diet, growth, development, children, adolescents.

Arona DB, Pietropaolo GG, Franco JVA. La dieta vegetariana en niños y preadolescentes. Evid Actual Pract Ambul. 2019;22(2):e002013.

\section{Escenario clínico}

Un médico de familia atiende a una niña de 8 años, sana, sin antecedentes relevantes y examen físico normal, que viene acompañada por su madre. La niña refiere que quiere iniciar una dieta vegetariana, pero la madre se pregunta si esto puede causar algún impacto en su crecimiento.

\section{Pregunta que generó el caso}

¿La dieta vegetariana (exposición), causa algún tipo de impacto en el crecimiento (resultado) de los niños y preadolescentes (población) en comparación con los que tienen una dieta omnívora (comparación)?

\section{Estrategia de búsqueda}

Se realizaron dos búsquedas en Pubmed en Junio de 2019. En una de ellas, se emplearon palabras claves como "vegetarian children", "vegetarian diets", "effects on growth vegetarian child". Se limitó la búsqueda empleando los filtros "systematic review", y se seleccionó una revisión sistemática ${ }^{1}$. La otra búsqueda, se realizó utilizando la estrategia: ((Diet, Vegetarian[Mesh]) AND Growth[Mesh]) AND Adolescent[Mesh], y se seleccionó un estudio longitudinal ${ }^{2}$.

\section{Algunos datos sobre las dietas vegetarianas}

Una persona vegetariana es aquella que se abstiene de comer carne, pescado, mariscos y productos elaborados con los mismos, pudiendo o no incluir otros derivados animales como lácteos, huevos o miel. Esta práctica, se pueden clasificar según los alimentos que se aceptan, en distintas categorias ${ }^{3}$ (ver Tabla 1).

La popularidad de las dietas vegetarianas está en aumento sobre todo en las edades pediátrica y adolescente, debido a que miembros de la familia ya son vegetarianos o, a veces, por iniciativa propia. Ambas situaciones suponen un desafío para los profesionales de la salud, que generalmente no han recibido formación adecuada durante sus estudios de medicina sobre este tema ${ }^{4}$

Tabla 1. Clasificación de las dietas vegetarianas

\begin{tabular}{|l|l|}
\hline Denominación & \multicolumn{1}{c|}{ Tipo de alimentos incluidos } \\
\hline Ovo-Lacto-Vegetariana & Productos de origen vegetal, huevos y lácteos. \\
Ovo-Vegetariana & $\begin{array}{l}\text { Productos de origen vegetal y huevos. } \\
\text { Productos de origen vegetal, leche y sus derivados }\end{array}$ \\
Lacto-Vegetariana & $\begin{array}{l}\text { Solo consume alimentos de origen vegetal. No acepta productos que hayan implicado sufrimiento } \\
\text { o explotación de algún animal. }\end{array}$ \\
Vegana & $\begin{array}{l}\text { Más del } 70 \% \text { de los alimentos crudos o cocidos a menos de } 42^{\circ}, \text { manteniendo atributos que se } \\
\text { pierden a mayores temperaturas. }\end{array}$ \\
Frugívora & Únicamente frutos, crudos y de estación. \\
\hline
\end{tabular}

\footnotetext{
a Departamento de Salud (Carrera de Medicina), Universidad Nacional de La Matanza. danielabelenarona@hotmail.com

b Departamento de Investigación, Instituto Universitario Hospital Italiano.

c Departamento de Salud, Universidad Nacional de La Matanza.

d Servicio de Medicina Familiar y Comunitaria, Hospital Italiano de Buenos Aires. juan.franco@hospitalitaliano.org.ar
} 


\section{Resumen de la evidencia}

\section{Schürmann S, et al. Vegetarian diets in children: a systematic} review. Eur J Nutr. 2017;56(5):1797-1817

El objetivo de esta revisión sistemática fue evaluar los estudios existentes sobre la ingesta dietética y el estado nutricional de los niños y adolescentes vegetarianos. Se realizó una búsqueda en MEDLINE, Web of Science y Google Académico, y se obtuvieron 24 artículos relevantes, publicados de 1988 a 2013, desarrollados en Europa, América del Norte, Australia y Nueva Zelanda. Las muestras de los estudios utilizados cubrieron los rangos etarios de 0 a 18 años, de sujetos de ambos sexos, sanos. En cuanto al tipo de alimentación, se dividieron en dietas de tipo vegetariana, dieta ovo-lacto-vegetariana, adventistas del séptimo día con patrones dietarios de tipo vegetarianos, y un grupo control formado por omnívoros, de igual edad y sexo. Se analizaron datos como el crecimiento a partir de los índices antropométricos (peso, talla, índice de masa corporal [IMC]), biomarcadores como proteínas, hierro, hormonas, perfil lipídico, ácidos grasos, marcadores óseos, y la ingesta de micronutrientes.

Los resultados del estudio fueron heterogéneos. Centrándonos en las dietas vegetarianas, se pudo observar que el desarrollo físico (altura, peso corporal, índice de masa corporal (IMC)) y sexual fue similar al grupo control. En cuanto a la ingesta de micronutrientes, los resultados fueron diversos: la ingesta de folato en vegetarianos fue superior, mientras que el consumo de vitamina $D$ fue menor. Los marcadores de formación ósea como la osteocalcina, el péptido C-terminal de tipo colágeno, y la fosfatasa alcalina ósea, fueron 10 a $20 \%$ más bajos en vegetarianos. Sin embargo, el perfil lipídico fue más favorable en los vegetarianos, con valores de colesterol total, triglicéridos y LDL significativamente más bajos. Por otro lado, las ingestas de energía y proteínas alcanzaron los valores de referencia. En contraparte, se observó una mayor prevalencia de deficiencia de hierro en los vegetarianos. Dentro de las limitaciones se evidenció en primer lugar una heterogeneidad en la composición y la evaluación de los patrones dietarios en los estudios incluidos. En segundo lugar, se incluyeron estudios de países desarrollados (limitando la generalizabilidad de los resultados) y no se realizó ningún tipo de muestreo probabilístico, con el posible sesgo de participación de voluntarios saludables (healthy user bias). Finalmente, la mitad de los estudios en esta revisión fueron de corte transversal y no permitieron obtener conclusiones sobre la asociación causal entre el crecimiento a largo plazo, el desarrollo y salud durante la infancia y la adolescencia.

\section{Movassagh EZ, et al. Vegetarian-style dietary pattern during adolescence has long-term positive impact on bone from adolescence to young adulthood: a longitudinal study. Nutr J. 2018;17(1):36}

El objetivo de este estudio longitudinal fue examinar la asociación entre los patrones dietarios, principalmente vegetarianismo, y las mediciones óseas desde la adolescencia hasta la edad adulta temprana. Para ello se reclutaron entre 1991 y 1993, en Canadá, datos de 125 participantes (53 mujeres) para el análisis de adolescentes (edad 12,7 \pm 2 años) y 115 participantes (51 mujeres) para el análisis de adultos (edad 28,2 \pm 3 años), que fueron estudiados con seguimientos anuales hasta 2011. Lo que se estudió fue el contenido mineral óseo (BMC) y la densidad mineral ósea del área (aBMD) del cuerpo total (TB), el cuello femoral (FN) y la columna lumbar (LS) que se midieron utilizando la densitometría de rayos $X$ de energía dual. T ambién fueron evaluados la antropometría, la ingesta dietética y la actividad física.

La evaluación de la ingesta dietética se realizó en distintos grupos dietarios: estilo vegetariano, estilo occidental, alto en grasa y valor proteico, mixto y snack. Se realizaron de dos a cuatro diarios alimentarios de 24 hs durante un año, y se analizaron utilizando un software de evaluación nutricional: Nutritional Assessment System, para estimar la ingesta diaria total de energía y nutrientes.

El resultado de este estudio lineal documentó, luego de controlar por covariables (sexo, edad, estatura, peso, puntaje de actividad física, consumo total de energía, velocidad de altura máxima), que el patrón dietario vegetariano fue adecuado en las variables de crecimiento óseo en adolescentes y adultos jóvenes.

Es importante destacar que la principal limitación de este estudio fue que su tamaño muestral pequeño, que no permitió realizar el análisis separado para mujeres y hombres ni evaluar la relación con otras variables como el patrón dietario para adultos jóvenes, el estado de fumador, el uso de anticonceptivos orales o el historial reproductivo (en mujeres). Como fortaleza se destaca el diseño longitudinal, que permite la observación a lo largo del tiempo del impacto de los distintos patrones dietarios en el crecimiento. Además, fue el primer estudio que evaluó patrones dietarios durante la adolescencia en asociación con la salud ósea en adultos jóvenes. Otro punto fuerte fue la recolección de los datos de la ingesta dietética utilizando múltiples observaciones de 24 horas durante un año, lo que se prefiere en vez de los cuestionarios de alimentación utilizados por otros estudios.

\section{Comentario}

Luego de analizar ambos estudios podríamos concluir que si bien hay una gran heterogeneidad en los resultados, una dieta vegetariana bien planificada sería saludable. Sin embargo, no debemos perder de vista que las personas expuestas a este tipo de alimentación tienen mayor riesgo de déficit de componentes esenciales, como el hierro, la vitamina B12, los ácidos grasos omega 3 , el calcio, la vitamina $D$ y el zinc ${ }^{5}$, si no los controlamos de cerca, aunque la deficiencia de estos micronutrientes se consideran resultados subrogados con un impacto clínico incierto. Tanto la Sociedad Americana de Pediatría ${ }^{6}$ y la Academia de Nutrición Dietética ${ }^{7}$ defienden las dietas vegetarianas adecuadamente planificadas como saludables y nutricionalmente apropiadas para este grupo etario.

Aunque la confianza en la evidencia disponible es limitada, la mayoría de los estudios analizados no mostraron efectos perjudiciales en niños y adolescentes. Incluso, apuntaron a resultados beneficiosos tales como: un desarrollo físico adecuado, menor riesgo de sobrepeso, perfil lipídico dentro de rangos normales, menor tendencia al desarrollo de enfermedades cardiovasculares o metabólicas.

Al recibir en la consulta a la paciente que concurre con su madre y plantea el deseo de una dieta vegetariana, el rol del médico es escuchar con empatía, acompañar en la decisión, brindar espacio para las dudas. También, debemos tranquilizar a la madre, asegurándole que su hija no presentaría ningún problema nutricional ni del crecimiento siguiendo una alimentación vegetariana equilibrada, reemplazando las proteínas y otros nutrientes de origen animal por alimentos de origen vegetal.

A pesar de que no existe ninguna evidencia científica que avale específicamente qué estudios hay que realizarle a una persona vegetariana, sería importante evaluar la presencia de anemia ferropénica y la deficiencia de B12, el componente más precario de estas dietas, dado que se encuentran únicamente en alimentos de origen animal. Por tal motivo, la Asociación Canadiense de Pediatría recomienda suplementar con esta vitamina a embarazadas vegetarianas, veganos y vegetarianos con déficit de vitamina $\mathrm{B} 12^{8}$.

Para finalizar la consulta, es importante planificar junto a la familia cómo será el abordaje de la dieta, debido a que la provisión 
de alimentos a esta niña, por su edad, es realizada por los adultos a cargo. Además, debemos tener en cuenta que está inserta en un contexto escolar, donde la alimentación provista podría no contemplar las restricciones de este tipo de dietas. Finalmente, es importante evaluar el contexto socioeconómico de la paciente y su familia, dado que la mayoría de los estudios sobre el tema fueron realizados en países desarrollados.

\section{Conclusiones de los comentadores}

La dieta vegetariana sería una alternativa saludable en niños y preadolescentes, aunque condicionaría un riesgo menor de déficit de ciertos micronutrientes. Se debería discutir esta evidencia con la familia, considerando aspectos prácticos de la aplicabilidad en el entorno de la paciente y supervisando la adecuada ingesta de micronutrientes.

\section{Referencias}

1. Schürmann S, Kersting M, Alexy U. Vegetarian diets in children: a systematic review. Dr. Dietrich Steinkopff Verlag GmbH and Co. KG; 2017 . Available from: 10.1007/s00394-017-1416-0.

2. Movassagh EZ, Baxter-Jones ADG, Kontulainen S, Whiting S, Szafron M, Vatanparast H. Vegetarian-style dietary pattern during adolescence has long-term positive impact on bone from adolescence to young adulthood: a longitudinal study. Nutrition journal. 2018;17(1):36. Available from: 10.1186/s12937-018-0324-3.

3. Agnoli C, Baroni L, Bertini I, Ciappellano S, Fabbri A, Papa M, et al. Position paper on vegetarian diets from the working group of the Italian Society of Human Nutrition. Nutrition, Metabolism and Cardiovascular Diseases. 2017 dec;27(12):1037-1052. Available from: 10.1016/j.numecd.2017.10.020.

4. Biarge MM, García RG. Niños vegetarianos, ¿niños sanos? Viernes 17 de febrero de 2017 Seminario: Niños vegetarianos, ¿niños sanos? 2017;p. 253-268.

5. Murray RS, Ridner E, Basile R, Munner M, Rovirosa A, Sanchez MA, et al. Alimentación vegetariana - Posición de la Sociedad Argentina de Nutrición. http://wwwsanutricionorgar/files/upload/files/Alimentacion\{_\}Vegetariana\{_\}Posicion\{_\}SANpdf. 2014;

6. Kleinman RE. Pediatric nutrition handbook, 6th edition - American Academy of Pediatrics; 2009.

7. Craig WJ, Mangels AR, Association AD. Position of the American Dietetic Association: vegetarian diets. Journal of the American Dietetic Association 2009 jul;109(7):1266-82. Available from: 10.1016/j.jada.2009.05.027.

8. Amit M. Vegetarian diets in children and adolescents. Paediatrics child health. 2010;15(5):303-14. 